Vietnam Journal of Mechanics, VAST, Vol.41, No. 4 (2019), pp. 287-300

DOI: https://doi.org/10.15625/0866-7136/13710

\title{
ON THE EFFECTIVE VISCOSITY OF FRESH CONCRETE: A HOMOGENIZATION APPROACH
}

\author{
Tuan Nguyen-Sy*, Duong Nguyen-The \\ Duy Tan University, Da Nang, Vietnam \\ ${ }^{*}$ E-mail: stuan.nguyen@gmail.com
}

Received: 27 March 2019 / Published online: 13 December 2019

\begin{abstract}
Two new homogenization schemes together with the classical generalized selfconsistent scheme (GSC) and its extension are proposed to deal with the effective viscosity of fresh concrete. All these models exploit the composite sphere morphology of suspension made of a viscous fluid and spherical particles. They differ from each other by the ways the extra fluid zone (EFZ) located in between the composite sphere are treated. The comparison with experimental data shows that the GSC provides very good result for a well arranged particle size scale that allows mapping the whole medium by composite spheres of different size scales. However, the GSC cannot be used for a suspension with a non negligible volume of the EFZ. For such a case, extensions of the GSC those take into account the contribution of the EFZ to the overall viscous behavior of the system is necessary to fit with experimental data. Two of them work very well for non-cohesive particles and the other can be employed for suspension with cohesive particles such as the case of fresh cement paste.
\end{abstract}

Keywords: homogenization, viscosity, fresh concrete, coated morphology.

\section{INTRODUCTION}

The knowledge of the viscosity of fresh concrete is very important for modeling different construction processes such as the prediction of the pumping pressure [1], simulation of the capacity of concrete to pass through the reinforcement grid of deep elements [2], etc. Fresh concrete can be considered as a suspension of gravel in fresh cement paste that is in turn a suspension of partially hydrated cement particles in water. Due to an extreme contrast between the constituents and their mobility, the overall viscosity of such a mixture is highly sensitive to the microstructure including the volume fraction, the shape and the spatial distribution of the phases. Here we precise that the static viscosity, i.e. the viscosity at zero-frequency loading, is considered.

In a seminal study, Einstein was the first who developed an analytical formula to relating the viscosity of a suspension to its particle volume fraction [3]. His theoretical result is limited to suspension containing a very low volume fraction of mono micro size spherical particles of which the interaction between the particles and the effect of

(C) 2019 Vietnam Academy of Science and Technology 
the transition zone between particles and fluid are negligible. Since Einsteins study, numerous experimental studies followed by empirical formulas have been developed for suspensions at high range of particle volume fraction [4,5], bi-modal and multi-modal particle sizes [6-8], divers shape [9-11] and nano particles [12-14]. Following experimental studies, theoretical concepts are also largely developed to deal with the effective viscosity of suspensions. Belong them, the homogenization theory is the most appropriate method to take into account the effect of microstructure of the composite to its overall viscosity. It is well-known that the homogenization method of elasticity can be employed to model the effective viscosity of a suspension thank to the analogy between linear elasticity and linear viscous behaviors [15]. Eshelby [16] based homogenization schemes such as dilute scheme or Mori-Tanaka $[17,18]$ scheme or the generalized selfconsistent scheme (GSC) $[19,20]$ can be directly employed to deal with a suspension containing a small or average volumetric fraction of particles. For a high concentration of mono-size particles, close to the percolation state, a modified generalized self-consistent scheme, that takes into account the binding fluid that is located inside the percolated particle packing, appears to be appropriate at least for a mono-size problem [21]. Multi-scale homogenization technique can be employed to deal with a well defined scale separation of particle size $[22,23]$. The later requires a sophisticated concept of percolated particle packing [24-26]. For example, $\mathrm{Vu}$ [27] measured the effective viscosity of suspensions containing two sizes particles and calibrated percolated packing parameters to fit the generalized self-consistent scheme with experimental data.

Fig. 1 shows a comparison between the largely used empirical formula of Krieger and Dougherty [5], the theoretical GSC scheme $[19,20]$ and experimental data measured

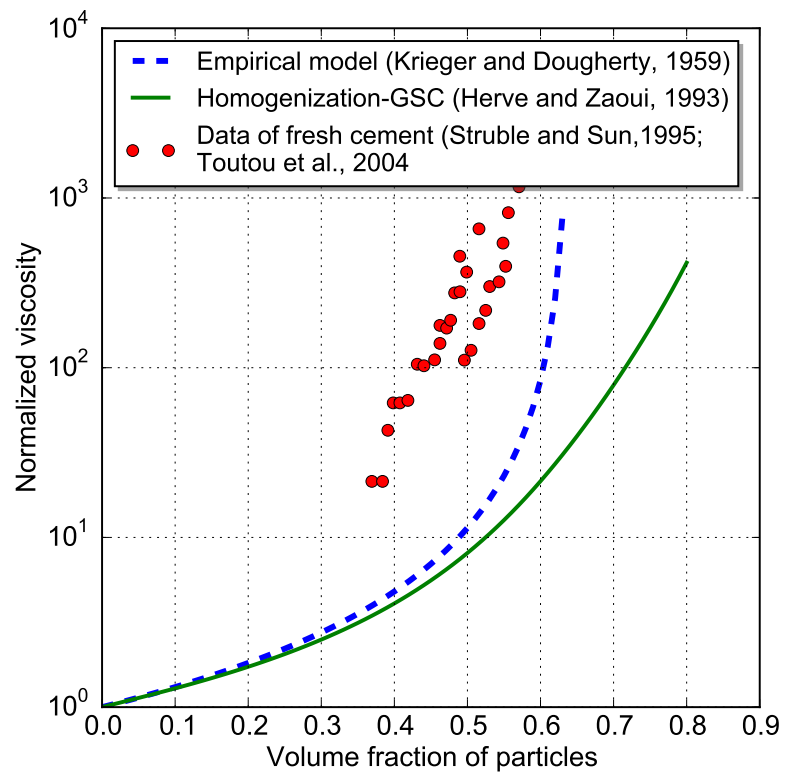

Fig. 1. Viscosity of fresh cement versus the volume fraction of particles: a comparison between experimental data and existent models 
on fresh cement by Struble and Sun [28] and Toutou et al. [22]. It is clear that both models are not appropriate for fresh cement. Indeed, Struble and Sun [28] and Toutou et al. [22] tried to fit the Krieger and Dougherty's formula with their data and they need to totally modify, case by case, the values of the parameters $[\eta]$ and $\phi_{M}$. Of cause such modification can allow fitting the data but it work for case by case and more importantly the calibrated parameters have no physical meaning. For example, Struble and Sun [28] obtained values $[\eta]=5$ to 6 while the theoretical value is $[\eta]=5 / 2[5]$.

Indeed, most of the existent empirical and theoretical models are limited to the situation of non cohesive particles, i.e. the solid particles are not pasted together when the volume fraction of particle is below the maximal value of the particle packing $\phi_{M}$. In other words, each single particle is isolated by the fluid phase if the particle volume fraction is smaller than $\phi_{M}$. Such assumption may not appropriate for fresh cement of which the partially hydrated cement particles may locally pasted together if they are placed closely in the space as illustrated by Fig. 2. Such coating morphology can form composite particles in which a part of the fluid content is blocked and becomes immobile. More precisely, part of the fluid content may act by the similar ways as the solid content that increases the viscosity of the whole medium.

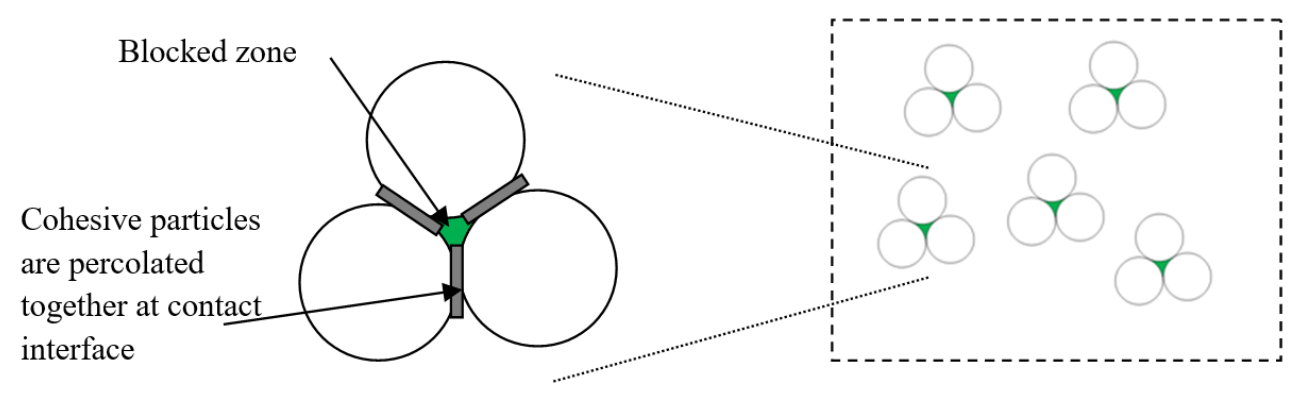

Fig. 2. Assumption of blocked fluid zone for the case of cohesive particles

For the best of our knowledge, no appropriate homogenization scheme exists in literature for predicting the effective viscosity of suspension containing cohesive particles as described by Fig. 2. Then it is of a great interest to develop solutions for such a problem to deal with fresh concrete and other similar materials.

This paper is dedicated to develop closed form solution for effective viscosity of fresh concrete on the basis of the homogenization theory. It is organized as following: first, the theoretical basis of the homogenization method and a review of existent homogenization method for effective viscosity of suspension are presented in Section 2. In Section 3, two appropriate homogenization models are developed to adapt with the morphologies of fresh concrete at both cement and gravel scales. These models are calibrated and validated against experimental data available in literature. Some important conclusions are given at the end of the paper. 


\begin{tabular}{cl} 
& \multicolumn{1}{c}{ NOTATIONS } \\
$\mu$ & Shear viscosity \\
hom & Index stands for a homogenized/effective value \\
$\mathrm{B}$ & Strain rate localization factor of a multi inclusion problem \\
$\phi$ & Volume fraction of particles
\end{tabular}

\section{THE BASIC OF THE HOMOGENIZATION THEORY AND A REVIEW OF EXSITENT MODELS}

A suspension can be regarded as a matrix-inclusion system. The classical dilute scheme [3] and Mori-Tanaka scheme [17] can be employed for a small to medium amount of particles. For a large volume fraction of particles, it is necessary to consider the mixture with more complex microstructure concept of the generalized self-consistent scheme that was previously developed for an equivalent elastic problem $[19,20]$.

\subsection{The basis of the homogenization theory}

The homogenization method consists to estimate the effective shear viscosity of a suspension by considering a special weighted average over its representative elementary volume (REV) such as

$$
\mu_{\text {hom }}=\langle\mu B\rangle,
$$

where the notation $\langle$.$\rangle stands for a volumetric average over the REV; \mu$ the viscosity of the constituents in the mixture; $B$ the strain rate localization factor that relates the local deviatoric strain rate $\dot{\varepsilon}_{D}$ and the volumetric average of the deviatoric strain rate (macroscopic deviatoric strain rate) $\left\langle\dot{\varepsilon}_{D}\right\rangle$ such as $\dot{\varepsilon}_{D}=B\left\langle\dot{\varepsilon}_{D}\right\rangle$. The localization factor $B$ must satisfy the conditions: $\langle B\rangle=1$. The viscosity of the matrix fluid and the volume fraction of the solid particles are supposed to be known so the problem reduces to finding the localization factor $B$ for both the particles inclusion and fluid matrix.

\subsection{The classical GSC scheme}

The GSC scheme consists to map the suspension containing spherical particles by a system of composite sphere that is then simplified to a problem of a single coated sphere in an infinite matrix of which the viscosity is the effective viscosity of the initial suspension. For an equivalent elastic problem, analytical solution of the effective shear modulus is first given by Christensen and Lo [19]. The analogy between a linear elastic problem and a linear viscous problem allows using that solution to predict the effective shear viscosity of a composite. For the case of suspension of particles in a viscous fluid with a viscous Poisson ratio equals to $v=1 / 2$, similar mathematical developments of the elastic problem can be realized with attention to avoid the division to the term $(1-2 v)$ that equal to zero in this case. Following analytical solution for the effective viscosity can be obtained after some mathematical manipulations

$$
\frac{\mu_{G S C}}{\mu_{f}}=\frac{-b_{G S C}-\sqrt{b_{G S C}^{2}-4 a_{G S C} c_{G S C}}}{a_{G S C}},
$$


where $\mu_{f}$ is the shear viscosity of fluid. The parameters $a_{G S C}, b_{G S C}$ and $c_{G S C}$ are defined by $a_{G S C}=-8(\delta-1)^{4}\left(4 \delta^{6}+16 \delta^{5}+40 \delta^{4}+55 \delta^{3}+40 \delta^{2}+16 \delta+4\right)$, $b_{G S C}=-2(\delta-1)\left(8 \delta^{9}+8 \delta^{8}+8 \delta^{7}+208 \delta^{6}+208 \delta^{5}-128 \delta^{4}-128 \delta^{3}-3 \delta^{2}-3 \delta-3\right)$,

$c_{G S C}=48 \delta^{10}+200 \delta^{7}-336 \delta^{5}+225 \delta^{3}+38$,

with $\delta^{3}=\phi$, in which $\phi$ is the volume fraction of the particles. For a suspension with a small amount of particles, i.e. $\phi \leq 1$, the series expansion of the solution (2) in the vicinity of zero yields

$$
\frac{\mu_{G S C}}{\mu_{f}} \approx 1+2.5 \phi+2.5 \phi^{2}
$$

It is of interest to note that the first two terms on the right side of Eq. (4) corresponds to the Einstein's model. Fig. 3 shows the evolution of the normalized effective viscosity $\mu_{G S C} / \mu_{f}$ estimated by the GSC (Eq. (2)) together with its second order series expansion (Eq. (4)) in comparison with Einstein's solution (that corresponds to the first order series expansion of GSC solution). The simple first order approximation can be employed for a suspension with smaller than $5 \%$ in volume of particles and the second order approximation and be used for less than $10 \%$ of particles.

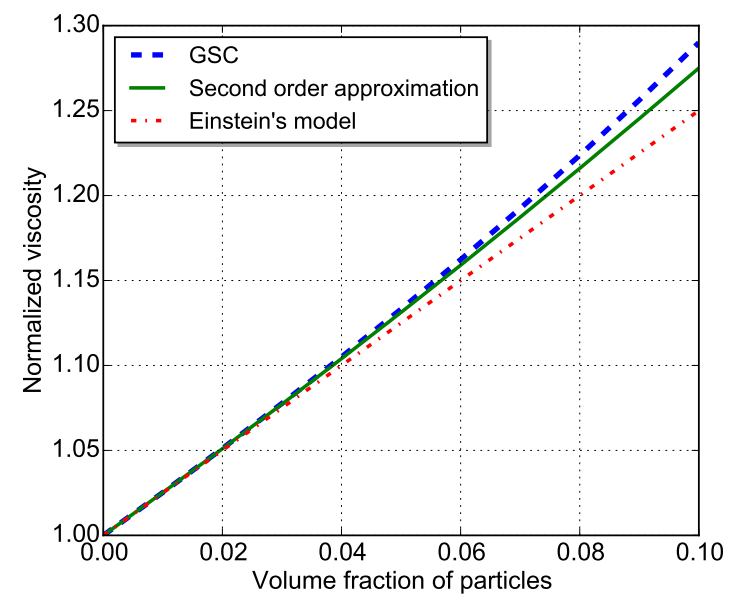

Fig. 3. Effective viscosity of suspension estimated by the GSC scheme and its series expansions

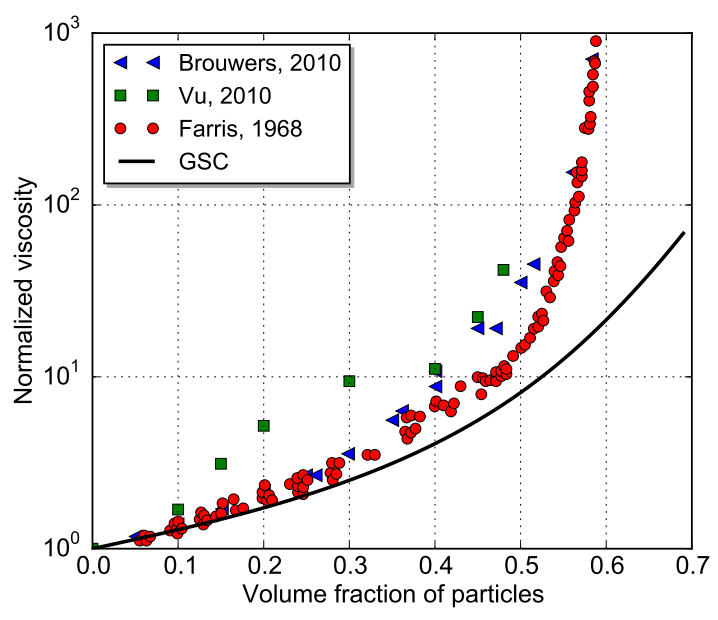

Fig. 4. A comparison between the generalized self-consistent scheme and data collected from different sources in literature

A comparison with experimental data shows also that for a low ranges of particle volume up to $30 \%$, the GSC fit very well with experimental data collected from different sources on mono-disperse suspension [8, 26, 29] (Fig. 4). However the GSC underestimates the effective viscosity at a higher volume fraction of particles.

It is important to remark that the GSC scheme ignores the extra fluid zone (EFZ) located in-between the coated spheres (Fig. 5(a)). Therefore this method is appropriate for 
an ideally arranged particle sizes of which small particles filled in between big particles to reduce the volume fraction of the EFZ to zero (Fig. 5(b)). But it is the main reason of the under-estimation of the GSC scheme for the case of mono-disperse suspension at high particle volume fraction as shown on Fig. 4. Therefore additional homogenization concept for the EFZ is necessary to improve the GSC scheme.
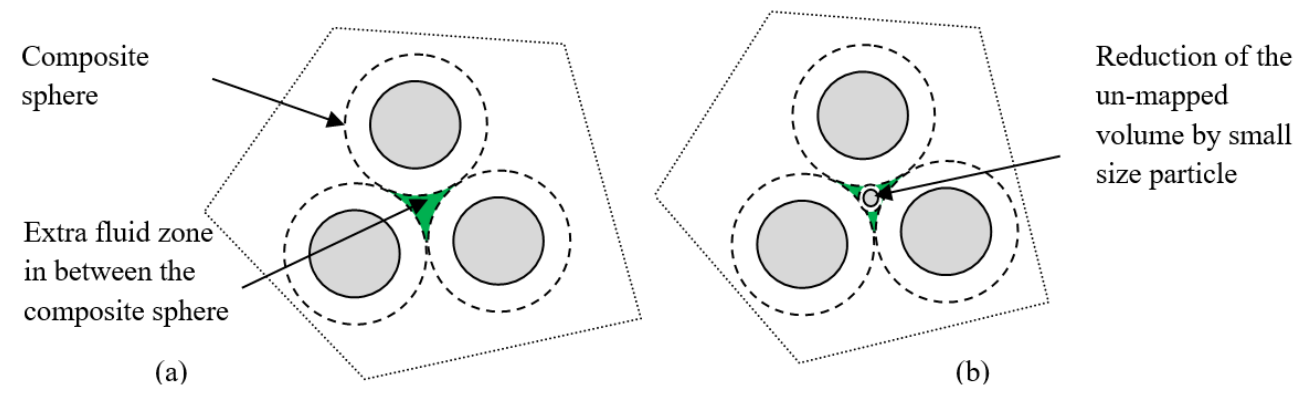

(b)

Fig. 5. Composite sphere mapping of suspensions containing spherical particles

\subsection{An improvement of the GSC considering the EFZ}

Marcadon et al. [21] modeled the effective elastic properties of composites those have the same microstructure of the considering suspension. Such model is straightforward for the present problem thank to the analogy between the linear elastic and linear viscous behaviors (as previously mentioned). The localization strain rate in the EFZ is obtained from the Eshelby's solution [16] while the localization strain rate in the composite spheres made of the particles and the coated fluid phase is given by Herve and Zaoui [20].

It is important to remark that in the solution of the average local strain derived by Herve and Zaoui [20] for a phase located in a composite sphere (equation 36 of their paper), there is a division to $(1-2 \mu)$ that equal to zero for the case of fluid because the Poisson ratio $\mu$ of fluid is $1 / 2$. Therefore some technical treatments are necessary when using their solution. For example, the Poisson ratio of fluid can be taken a little bit smaller than $1 / 2$ to avoid division to zero. By a more rigorous ways, the development of Herve and Zaoui [20] needs to be redeveloped in a ways that allows avoiding the division to the term $(1-2 \mu)$ that equals to zero. One the localization factors of all the phases are known, the effective viscosity of the mixture can be computed by Eq. (1). Analytical solution of the effective viscosity issues from this combination concept is a lot more cumbersome comparing to the solution (2) and could not be presented in this paper.

Consider for example the case of mono disperse of which the volume fraction of the EFZ is $36 \%$ and the volume fraction of the composite sphere is $64 \%$. A comparison with measured data shows that this concept (that is named by concept 1) reproduces very well the experimental observation (Fig. 6). It is a lot better than the GSC at high volume fraction of particles. We compare also the results obtained by the homogenization concept 1 with the famous empirical formula of Krieger and Dougherty [5] (KD) using the parameters $\phi_{\max }=0.64$ (that correspond to the maximum volume fraction of the solid phase of a random close packing of mono size sphere) and $[\eta]=2.5$. We have $\phi_{\max }=$ 
$1-V_{E F Z}$ with $V_{E F Z}$ the volume fraction of the EFZ. We observe that if the value $\phi_{\max }=$ 0.64 for the case of mono disperse of spherical particle is used, the KD method under estimate the effective viscosity of suspension close to percolation state. In literature, a value $\phi_{\max } \approx 0.57$ that is smaller than the theoretical value 0.64 is usually considered for the empirical KD method to fit with experimental data of mono-disperse suspension.

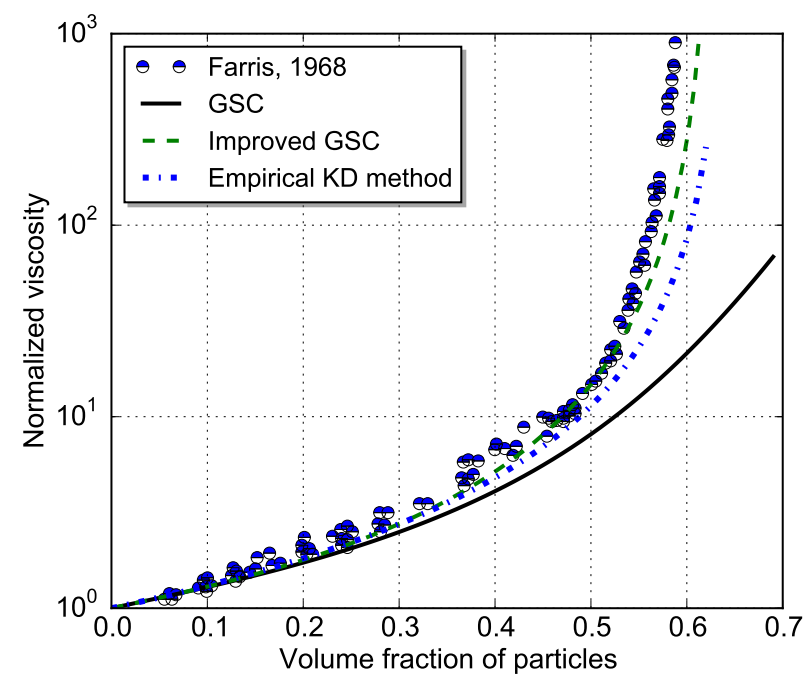

Fig. 6. The improved GSC scheme: a comparison with the GSC, data and the empirical KD method

\section{APPROPRIATE HOMOGENIZATION METHODS FOR FRESH CEMENT AND CONCRETE}

The effective viscosity of fresh concrete is modeled in two scales: the cement scale and the gravel scale. At the first scale, it is necessary to model the effective viscosity of fresh cement as function of the volume fraction of cement particles. Here we assume that the volume fraction of the particles is given, i.e. the evolution of the volume of the solid phase due to hydration is out of the scope of the present paper. At the second scale, the effective viscosity of fresh concrete is modeled assuming the viscosity of the cement phase as well as the volume fraction of gravels are given.

\subsection{The first scale: fresh cement}

As previously described by Fig. 2, cement particles are assumed to not suspending separately in fluid but they locally pasted together, event at small or medium volume fraction, to form alternative composite particles those block inside a part of the fluid phase. Therefore, a suspension with cohesive particles of volume fraction $\phi$ can be considered as a suspension with alternative particles of volume fraction $\phi+\phi_{b}$, where $\phi_{b}$ the total volume of the blocked fluid. Theoretically, $\phi_{b}$ must be smaller or equal to $1-\phi_{\max }$ that is $0.36\left(\phi+\phi_{b}\right)$ for a packing of mono size sphere. 
It is important to remark that such assumption allows reducing the volume fraction of the EFZ to zero. Therefore the GSC can be employed for the mixture of alternative particles and remaining (non blocked) fluid. Fig. 7 shows that the results obtained by considering a minimum possible value of $\phi_{b}$ that is $\phi_{b}=0$ provides a lower bound of the experimental data. A maximum value $\phi_{b}=0.36\left(\phi+\phi_{b}\right)$, i.e. $\phi_{b}=0.56 \phi$, (with mono-size assumption for cement particles) seems provides a good fit with the experimental data.

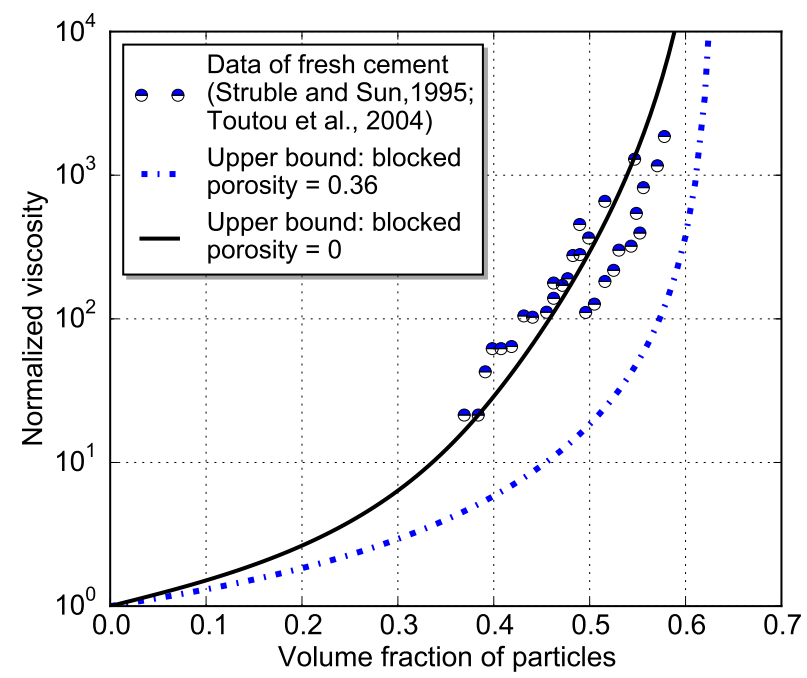

Fig. 7. Effective viscosity of cement: a comparison of the homogenization scheme and experimental data of fresh cement

\subsection{The second scale: fresh concrete}

A comparison with experimental data shows that the improved GSC scheme (IGSC), that was presented in Section 2.3, works very well for fresh concrete at the second scale that is a mixture of fresh cement and gravels (Fig. 8). That method assumes a spherical shape of the EFZ and employs the Eshelbys solution to estimate the local strain rate of the EFZ. However analytical results are very cumbersome.

Let consider a more simple assumption such that the average localization strain rate of the fluid phase located in the coated phase surrounding the gravel particles equal to that of the EFZ. This assumption allows using the solution of the localization factor obtained for the coated fluid phase of the composite sphere for the EFZ. We note by $B_{1}$ the localization factor of the particles and $B_{2}$ the localization factor of the coated fluid and the EFZ. Using Eq. (1), to calculate the effective viscosity of the suspension, we obtain

$$
\mu_{\mathrm{hom}}=\lim _{\mu_{p} \rightarrow \infty}\left[\phi \mu_{p} B_{1}+(1-\phi) \mu_{f} B_{2}\right]
$$

The closed-form solution to this problem is

$$
\frac{\mu}{\mu_{\mathrm{cement}}}=\frac{-b-\sqrt{b^{2}-4 a c}}{a}
$$




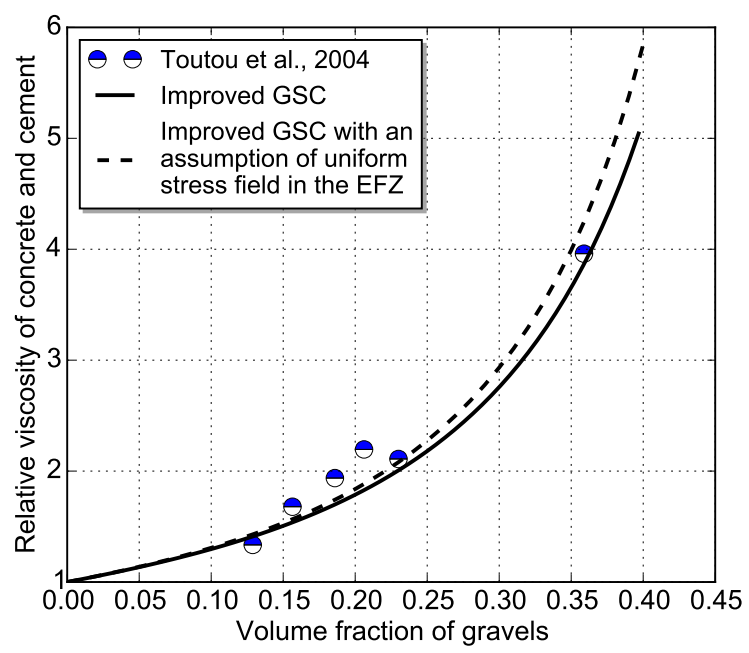

Fig. 8. Relative viscosity between concrete and cement versus the volume fraction of gravels: a comparison between the present model and data of Toutou et al. [22]

where the parameters $a, b$ and $c$ are defined by

$$
\begin{aligned}
a= & -\frac{24}{5}\left(\delta^{2}+\delta+1\right)(\delta-1)^{4}\left(4 \delta^{6}+16 \delta^{5}+40 \delta^{4}+55 \delta^{3}+40 \delta^{2}+16 \delta+4\right), \\
b= & -24 \delta^{3}(\delta-1)\left(2 \delta^{8}+4 \delta^{7}+6 \delta^{6}+13 \delta^{5}+20 \delta^{4}+13 \delta^{3}+6 \delta^{2}+4 \delta+2\right) \phi_{\max } \\
& +\frac{6}{5}(\delta-1)\left(32 \delta^{11}+64 \delta^{10}+96 \delta^{9}+36 \delta^{8}-24 \delta^{7}-28 \delta^{6}+168 \delta^{5}\right. \\
& \left.+339 \delta^{4}+174 \delta^{3}+9 \delta^{2}+6 \delta+3\right), \\
c= & 3 \delta^{3}\left[56 \delta^{3}(\delta+1)\left(\delta^{2}-1\right)+\left(16 \delta^{7}+19\right)\left(\delta^{2}+\delta+1\right)\right] \phi_{\max } \\
& -\frac{6}{5}(\delta-1)\left(16 \delta^{11}+32 \delta^{10}+48 \delta^{9}+88 \delta^{8}+128 \delta^{7}\right. \\
& \left.+56 \delta^{6}+84 \delta^{5}+187 \delta^{4}+122 \delta^{3}+57 \delta^{2}+38 \delta+19\right),
\end{aligned}
$$

with $\delta^{3}=\phi / \phi_{\max }$ and $\phi_{\max }=1-V_{E F Z}$. For the particular case with no EFZ zone, i.e. $V_{E F Z}=0$, the solution (6) is simplified to the solution (2) of the GSC. Note that $\phi_{\max }=$ 0.64 for a packing of sphere. A comparison of the solution (6) with experimental data of fresh concrete [22] as well as with the improved GSC presented in Section 2.3 is shown on Fig. 8. In this figure, the viscosity of fresh concrete is normalized to the viscosity of the fresh cement paste so the input parameters such as the viscosity of fluid and the porosity of concrete are not required for the simulation. The only parameter needed is the volume fraction of gravels. Of course, the viscosity of fluid is needed for determining the absolute viscosity of the mixture. Even though a very simplified assumption of the mechanical field in the EFZ is considered, the solution (6) fit very well with data and the model of Section 2.3. More importantly, solution (6) is explicit and then very easy to use in practice. 


\section{CONCLUSIONS}

Two homogenization concepts are proposed to model fresh concrete at two scales: fresh cement scale and gravel scale. At the first scale, fresh cement is regarded as a suspension of cohesive particles in viscous fluid. The cohesive particles are assumed to locally pasted together to form alternative particles, each of them is a particle packing containing blocked fluid. We consider a mono suspension and an ideal polydisperse suspension (such as the volume fraction of the blocked zone tends to zero) and we observed that the ideal polydisperse suspension provide an under bound while the mono size suspension correspond to a upper bound. Most of experimental data fit with the mono size assumption but some point lay in between the bounds.

At the second scale, the mixture between fresh cement and gravel is modeled by an improved GSC. Analytical solutions are derived considering a simplified assumption of uniform mechanical field in the extra fluid zone (EFZ). A mono size suspension for gravels is a good approximation and a comparison with experimental data show that such assumption allows the model to fit very well with data.

The workflow of the present homogenization model for the prediction of the effective viscosity of fresh concrete is resumed as following:

(1) Input: volume fraction of gravels $\phi_{g}$, volume fraction of cement particles $\phi_{c 0}$ and viscosity of fluid $\mu_{f}$.

(2) First scale: compute the relative volume fraction of cement in cement-fluid mixture (fresh cement) $\phi_{c}=\phi_{c 0} /\left(1-\phi_{g}\right)$ then compute the volume fraction of the local cement particle parkings $\phi_{c p}=\phi_{c} / 0.64$. Introducing the volume fraction $\phi_{c p}$ together with the input viscosity of the fluid phase $\mu_{f}$ in Eqs. (2) and (3) to compute the effective viscosity of fresh cement.

(3) Second scale: Once the viscosity of fresh cement is obtained from the first scale and the volume fraction of gravel particles is given by input data, we can compute the final effective viscosity of fresh concrete using Eqs. (6) and (7).

The dependence of effective viscosity on the thixotropic and curing behavior of cementbased materials are out of the scope of the present paper that focus on a homogenization method and the effect of the microstructure of the suspension.

\section{ACKNOWLEDGMENT}

This research is funded by Vietnam National Foundation for Science and Technology Development (NAFOSTED) under grant number 107.01-2016.17. The authors express deep thanks to Reviewers for their suggestions and comments.

\section{REFERENCES}

[1] A. Leemann and F. Winnefeld. The effect of viscosity modifying agents on mortar and concrete. Cement and Concrete Composites, 29, (5), (2007), pp. 341-349. https://doi.org/10.1016/j.cemconcomp.2007.01.004.

[2] H. Okamura and M. Ouchi. Self-compacting concrete. Journal of Advanced Concrete Technology, 1, (1), (2003), pp. 5-15. https://doi.org/10.3151/jact.1.5.

[3] A. Einstein. Effect of suspended rigid spheres on viscosity. Ann. Phys, 19, (1906), pp. 289-306. 
[4] M. Mooney. The viscosity of a concentrated suspension of spherical particles. Journal of Colloid Science, 6, (2), (1951), pp. 162-170. https://doi.org/10.1016/0095-8522(51)90036-0.

[5] I. M. Krieger and T. J. Dougherty. A mechanism for non Newtonian flow in suspensions of rigid spheres. Transactions of the Society of Rheology, 3, (1), (1959), pp. 137-152. https://doi.org/10.1122/1.548848.

[6] J. V. Robinson. The viscosity of suspensions of spheres. The Journal of Physical Chemistry, 53, (7), (1949), pp. 1042-1056. https://doi.org/10.1021/j150472a007.

[7] G. F. Eveson, S. G. Ward, and R. L. Whitmore. Classical colloids. Theory of size distribution; paints, coals, greases, etc. Discussions of the Faraday Society, 11, (1951), pp. 11-14. https://doi.org/10.1039/DF9511100011.

[8] R. J. Farris. Prediction of the viscosity of multimodal suspensions from unimodal viscosity data. Transactions of the Society of Rheology, 12, (2), (1968), pp. 281-301. https://doi.org/10.1122/1.549109.

[9] D. H. Berry and W. B. Russel. The rheology of dilute suspensions of slender rods in weak flows. Journal of Fluid Mechanics, 180, (1987), pp. 475-494. https://doi.org/10.1017/S0022112087001915.

[10] F. M. Van der Kooij, E. S. Boek, and A. P. Philipse. Rheology of dilute suspensions of hard platelike colloids. Journal of Colloid and Interface Science, 235, (2), (1987), pp. 344-349. https://doi.org/10.1006/jcis.2000.7336.

[11] I. Marti, O. Höfler, P. Fischer, and E. J. Windhab. Rheology of concentrated suspensions containing mixtures of spheres and fibres. Rheologica Acta, 13, (4), (2005), pp. 474-480. https://doi.org/10.1007/s00397-005-0432-9.

[12] X. Wang, X. Xu, and S. U. Choi. Thermal conductivity of nanoparticle-fluid mixture. Journal of Thermophysics and Heat Transfer, 13, (4), (1999), pp. 474-480. https://doi.org/10.2514/2.6486.

[13] C. T. Nguyen, F. Desgranges, G. Roy, N. Galanis, T. Maré, S. Boucher, and H. A. Mintsa. Temperature and particle-size dependent viscosity data for water-based nanofluids-hysteresis phenomenon. International Journal of Heat and Fluid Flow, 28, (6), (2007), pp. 1942-1506. https://doi.org/10.1016/j.ijheatfluidflow.2007.02.004.

[14] J. H. Lee, K. S. Hwang, S. P. Jang, B. H. Lee, J. H. Kim, S. U. Choi, and C. J. Choi. Effective viscosities and thermal conductivities of aqueous nanofluids containing low volume concentrations of Al2O3 nanoparticles. International Journal of Heat and Mass Transfer, 51, (11), (2008), pp. 2651-2656. https://doi.org/10.1016/j.ijheatmasstransfer.2007.10.026.

[15] Z. Hashin. Viscoelastic behavior of heterogeneous media. Journal of Applied Mechanics, 32, (3), (1965), pp. 630-636. https://doi.org/10.1115/1.3627270.

[16] J. D. Eshelby and R. E. Peierls. The determination of the elastic field of an ellipsoidal inclusion, and related problems. Proceedings of the Royal Society of London. Series A. Mathematical and Physical Sciences, 241, (1226), (1957), pp. 376-396. https://doi.org/10.1098/rspa.1957.0133.

[17] T. Mori and K. Tanaka. Average stress in matrix and average elastic energy of materials with misfitting inclusions. Acta Metallurgica, 21, (5), (1973), pp. 571-574. https://doi.org/10.1016/0001-6160(73)90064-3.

[18] Y. Benveniste. A new approach to the application of Mori-Tanaka's theory in composite materials. Mechanics of Materials, 6, (2), (1987), pp. 147-157. https://doi.org/10.1016/01676636(87)90005-6.

[19] R. M. Christensen and K. H. Lo. Solutions for effective shear properties in three phase sphere and cylinder model. Journal of the Mechanics and Physics of Solids, 27, (4), (1987), pp. 315-330. https://doi.org/10.1016/0022-5096(79)90032-2. 
[20] E. Herve and A. Zaoui. N-layered inclusion-based micromechanical modelling. International Journal of Engineering Science, 31, (1), (1993), pp. 1-10. https://doi.org/10.1016/00207225(93)90059-4.

[21] E. Herve and A. Zaoui. Micromechanical modeling of packing and size effects in particulate composites. International Journal of Solids and Structures, 44, (25), (2007), pp. 8213-8228. https://doi.org/10.1016/j.ijsolstr.2007.06.008.

[22] Z. Toutou, C. Lanos, Y. Mélinge, and N. Roussel. Modèle de viscosité multi-échelle: de la pâte de ciment au micro-béton. Rhéologie, 5, (2004), pp. 1-9.

[23] Z. Toutou and N. Roussel. Multi scale experimental study of concrete rheology: from water scale to gravel scale. Materials and Structures, 39, (2), (2004), pp. 189-199. https://doi.org/10.1617/s11527-005-9047-y.

[24] D. J. Durian. Foam mechanics at the bubble scale. Physical Review Letters, 75, (Dec, 1995), pp. 4780-4783. https://doi.org/10.1103/PhysRevLett.75.4780.

[25] N. Phan-Thien and D. C. Pham. Differential multiphase models for polydispersed suspensions and particulate solids. Journal of Non-Newtonian Fluid Mechanics, 72, (2), (1997), pp. 305318. https://doi.org/10.1016/S0377-0257(97)90002-1.

[26] T.-S. Vu, G. Ovarlez, and X. Chateau. Macroscopic behavior of suspensions of noncolloidal particles in yield stress fluids. Journal of Rheology, 54, (06, 2010). https://doi.org/10.1122/1.3439731.

[27] T. S. Vu. Rhéologie des suspensions non newtoniennes. PhD thesis, Université Paris-Est, (2010).

[28] L. Struble and G.-K. Sun. Viscosity of Portland cement paste as a function of concentration. Advanced Cement Based Materials, 2, (2), (1995), pp. 62-69. https://doi.org/10.1016/10657355(95)90026-8.

[29] H. J. H. Brouwers. Viscosity of a concentrated suspension of rigid monosized particles. Physical Review E, 81, (5), (2010). https://doi.org/10.1103/PhysRevE.81.051402.

\section{APPENDIX The mathematical development of the proposed closed form solutions}

The homogenization method consists to estimate the effective shear viscosity of a suspension by an average over its representative elementary volume (REV) (see Eq. (1)). To deal with a large range of particle concentration, it is preferred to adopt the concept of the generalized self-consistent homogenization theory $[19,20]$. Unfortunately, the analytical solutions developed by Herve and Zaoui [20] are not straightforward for the problem of suspension of solid particles in viscous fluid because of a fluid's Poisson ratio of $v_{f}=0.5$ that leads to several undefined terms containing a division to $\left(1-2 v_{f}\right)$. Their workflow can be followed but a major modification is necessary.

Indeed, following Herve and Zaoui [20], we consider a two phase concentric sphere, made of a rigid core and a linear viscous fluid coated layer, surrounded by an infinite homogeneous viscous matrix (Fig. 9). The rigid core and the coated phase of the coated inclusion are numbered by 1 and 2, respectively. The infinite matrix is the phase 3 .

Under a simple uniform macroscopic shear stress, the fluid velocity in the system has following components in spherical coordinates

$$
\begin{array}{ll}
v_{r}=V_{r}(r) \sin ^{2} \theta \cos 2 \phi, & v_{\theta}=V_{\theta}(r) \sin \theta \cos \theta \cos 2 \phi, \\
v_{\phi}=V_{\phi}(r) \sin \theta \sin 2 \phi, & V_{\phi}(r)=-V_{\theta}(r) .
\end{array}
$$




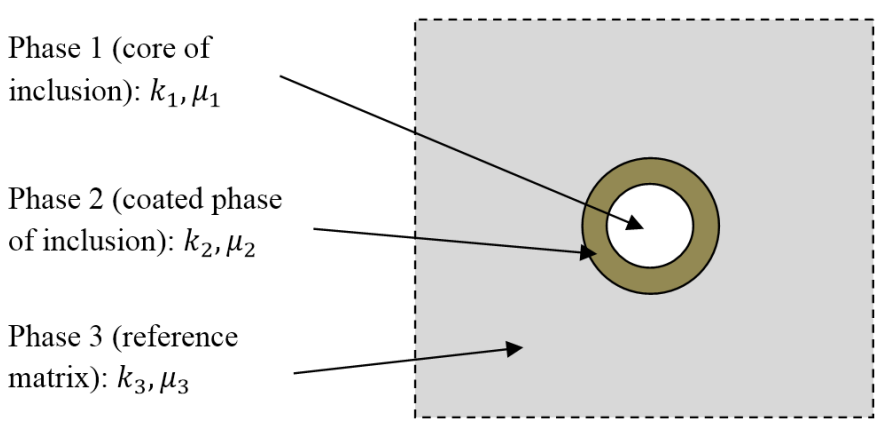

Fig. 9. Coated spherical inclusion in an infinite reference matrix

The correspondent strain rates are

$$
\begin{aligned}
& \dot{\varepsilon}_{r r}=V_{r}^{\prime} \sin ^{2} \theta \cos 2 \phi, \\
& \dot{\varepsilon}_{\theta \theta}=\frac{1}{r} V_{r} \sin ^{2} \theta \cos 2 \phi+\frac{1}{r} V_{\theta} \cos 2 \theta \cos 2 \phi, \\
& \dot{\varepsilon}_{\phi \phi}=\frac{2}{r} V_{\phi} \cos 2 \phi+\frac{1}{r} V_{r} \sin ^{2} \theta \cos 2 \phi+\frac{1}{r} V_{\theta} \cos ^{2} \theta \cos 2 \phi, \\
& \dot{\varepsilon}_{r \theta}=\frac{1}{2}\left(\frac{2}{r} V_{r}+V_{\theta}^{\prime}-\frac{1}{r} V_{\theta}\right) \sin \theta \cos \theta \cos 2 \phi, \\
& \dot{\varepsilon}_{r \phi}=-\frac{1}{2}\left(\frac{2}{r} V_{r}-V_{\phi}^{\prime}-\frac{1}{r} V_{\phi}\right) \sin \theta \sin 2 \phi, \\
& \dot{\varepsilon}_{\theta \phi}=-\frac{1}{r}\left(2 V_{\theta}+V_{\phi}\right) \cos \theta \sin 2 \phi .
\end{aligned}
$$

The volumetric strain rate is

$$
\operatorname{tr} \dot{\varepsilon}=\left(V_{r}^{\prime}+\frac{2}{r} V_{r}-\frac{3}{r} V_{\theta}\right) \sin ^{2} \theta \cos 2 \phi .
$$

The stresses components are: $\sigma_{j k}=2 \mu\left(\frac{v}{(1-2 v)} \operatorname{tr} \dot{\varepsilon}+\dot{\varepsilon}_{j k}\right)$ with $j, k=r, \theta, \phi$. The velocities $V_{r}$ and $V_{\theta}$, those are solutions of the combinations of the constitutive equation and the equilibrium equation, and the correspondent radial and tangent stress: $\left[V_{r}, V_{\theta}, S_{r r}^{(i)}, S_{r \theta}^{(i)}\right]^{t}$ $=L_{r}^{(i)}[A, B, C, D]^{t}$ with $i=1$ or 2 and

$$
L_{r}^{(i)}=\left[\begin{array}{cccc}
r & -\frac{6 v_{i}}{4 v_{i}-7} r^{3} & -\frac{3}{2 r^{4}} & \frac{5-4 v_{i}}{2\left(1-2 v_{i}\right)} \frac{1}{r^{2}} \\
r & r^{3} & \frac{1}{r^{4}} & \frac{1}{r^{2}} \\
2 \mu_{i} & \frac{6 v_{i}}{4 v_{i}-7} \mu_{i} r^{2} & \frac{12}{r^{5}} \mu_{i} & \frac{2\left(v_{i}-5\right)}{1-2 v_{i}} \frac{\mu_{i}}{r^{3}} \\
2 \mu_{i} & -2 \frac{7+2 v_{i}}{4 v_{i}-7} \mu_{i} r^{2} & -\frac{8}{r^{5}} \mu_{i} & \frac{2\left(v_{i}+1\right)}{1-2 v_{i}} \frac{\mu_{i}}{r^{3}}
\end{array}\right] .
$$


The strain rate concentration factors of the coated layer (see Eq. (1)) is

$$
\tilde{B}_{1}=\frac{A_{1}}{A_{3}}-\frac{B_{1}}{A_{3}} \frac{21 R_{1}^{2}}{5\left(4 v_{1}-7\right)},
$$

and

$$
\tilde{B}_{2}=\frac{A_{2}}{A_{3}}-\frac{B_{2}}{A_{3}} \frac{21 R_{2}^{2}}{5\left(4 v_{2}-7\right)} \frac{1-X^{5}}{1-X^{3}},
$$

where we noted the ratio between the radii of the inner core and the coated inclusion by: $X=R_{1} / R_{2} ; v_{1}$ and $v_{2}$ the Poissons ratios of the core and the coated phase of the inclusion, respectively. They are related to the bulk and shear moduli by the classical formula: $v_{i}=\left(3 k_{i}-2 \mu_{i}\right) /\left(6 k_{i}+2 \mu_{i}\right)$ with $i=1$ or 2 . The terms $A_{1} / A_{3}, A_{2} / A_{3}, B_{1} / A_{3}$ and $B_{2} / A_{3}$ are determined by

$$
\frac{A_{1}}{A_{3}}=\frac{P_{22}^{(2)}}{P_{11}^{(2)} P_{22}^{(2)}-P_{12}^{(2)} P_{21}^{(2)}}, \quad \frac{B_{1}}{A_{3}}=\frac{-P_{21}^{(2)}}{P_{11}^{(2)} P_{22}^{(2)}-P_{12}^{(2)} P_{21}^{(2)}},
$$

and

$$
\frac{A_{2}}{A_{3}}=\frac{P_{11}^{(1)} P_{22}^{(2)}-P_{12}^{(1)} P_{21}^{(2)}}{P_{11}^{(2)} P_{22}^{(2)}-P_{12}^{(2)} P_{21}^{(2)}}, \quad \frac{B_{2}}{A_{3}}=\frac{P_{22}^{(1)} P_{21}^{(2)}}{P_{11}^{(2)} P_{22}^{(2)}-P_{12}^{(2)} P_{21}^{(2)}}
$$

where the $[4 \times 4]$ matrix $\mathbf{P}^{(1)}$ and $\mathbf{P}^{(2)}$ are determined by

$$
\boldsymbol{P}^{(1)}=\boldsymbol{M}^{(1)} ; \boldsymbol{P}^{(2)}=\boldsymbol{M}^{(2)} \boldsymbol{M}^{(1)},
$$

with

$$
\boldsymbol{M}^{(i)}=\left(\boldsymbol{L}_{R_{i}}^{(i+1)}\right)^{-1} \boldsymbol{L}_{R_{i}}^{(i)}
$$

in which the matrix $\boldsymbol{L}_{r}^{(i)}$ is defined by Eq. (11).

The parameters $\tilde{B}_{1}$ and $\tilde{B}_{2}$ are functions of the elastic properties of the coated inclusion and the matrix phase as well as the ratio $X$ of the coated inclusion. It is of interest to remark that for the case of solid with $v_{1} \neq 0.5$ and $v_{2} \neq 0.5$, the solutions (12) and (13) are equivalent to the classical solution obtained by Herve and Zaoui [20]. However the solutions obtained herein are also valid for fluid with $v_{2}=0.5$. 